\title{
Geno- and phenotypic characteristics of a transfected Babesia bovis 6-Cys-E knockout clonal line
}

\author{
Heba F. Alzan ${ }^{1,2}$, Marta G. Silva', William C. Davis ${ }^{1}$, David R. Herndon ${ }^{3}$, David A. Schneider ${ }^{1,3}$ and Carlos E. Suarez ${ }^{1,3^{*}}$
}

\begin{abstract}
Background: Babesia bovis is an intra-erythrocytic tick-transmitted apicomplexan protozoan parasite. It has a complex lifestyle including asexual replication in the mammalian host and sexual replication occurring in the midgut of host tick vector, typically, Rhipicephalus microplus. Previous evidence showed that certain B. bovis genes, including members of 6-Cys gene family, are differentially expressed during tick and mammalian stages of the parasite's life cycle. Moreover, the 6-Cys E gene is differentially expressed in the T3Bo strain of B. bovis tick stages, and anti 6-Cys E antibodies were shown to be able to inhibit in vitro growth of the phenotypically distinct B. bovis Mo7clonal line.
\end{abstract}

Methods: In this study, the 6-Cys $E$ gene of B. bovis T3Bo strain was disrupted by transfection using a plasmid containing 6-Cys gene $E 5^{\prime}$ and $3^{\prime}$ regions to guide homologous recombination, and the egfp-bsd fusion gene under control of a ef-1a promoter, yielding a B. bovis clonal line designated 6-Cys EKO-cln. Full genome sequencing of 6-Cys EKO-cln parasites was performed and in vitro inhibition assays using anti 6-Cys E antibodies.

Results: Full genome sequencing of 6-Cys EKO-cln B. bovis demonstrated single insertion of egfp-bsd gene that disrupts the integrity of 6-Cys gene E. Undistinguishable growth rate of 6-Cys EKO-cln line compared to wild-type 6-Cys $E$ intact T3Bo B. bovis strain in in vitro cultures indicates that expression of gene 6-Cys $E$ is not essential for blood stage replication in this strain. In vitro inhibition assays confirmed the ability of anti- 6 Cys $E$ antibodies to inhibit the growth of the wild-type Mo7 and T3Bo B. bovis parasites, but no significant inhibition was found for 6Cys EKO-cln line parasites.

Conclusions: Overall, the data suggest that the anti-6 Cys E antibody neutralising effect on the wild type strains is likely due to mechanical hindrance, or cross-reactivity, rather than due to functional requirements of 6-Cys gene $E$ product for survival and development of the erythrocyte stages. Further investigation is underway to determine if the 6-Cys E protein is required for replication and sexual stage development of $B$. bovis during tick stages.

Keywords: B. bovis transfection, 6-Cys E gene, Cell sorting, Transfected clonal line

\section{Background}

Babesia bovis is an apicomplexan tick-borne parasite, mainly transmitted by Riphicephalus microplus, responsible for acute disease in bovines. The disease causes important economic loses in endemic areas worldwide, and improved methods of control are needed. A common

\footnotetext{
* Correspondence: ces@vetmed.wsu.edu

${ }^{1}$ Department of Veterinary Microbiology and Pathology, College of Veterinary Medicine, Washington State University, Pullman, WA, USA

${ }^{3}$ Animal Disease Research Unit, United States Department of Agriculture -

Agricultural Research Service, Pullman, WA, USA

Full list of author information is available at the end of the article
}

method used to prevent acute babesiosis in endemic areas, such as Australia, Argentina and Mexico, is the use of B. bovis attenuated vaccines, which have multiple limitations [1]. Subunit vaccines able to elicit sterile immunity or prevent acute babesiosis would be ideal prevention tools, but they remain unavailable. Additional tools for enhanced control include vaccines designed to block transmission of the parasites by the tick vector. Development of such vaccines requires the identification of relevant antigens expressed in the tick stages of the parasites. Recent work resulted in the identification of 
candidate antigens that can be used for the developing of Babesia transmission blocking vaccines [2], including the members of the 6-Cys gene family. The apicomplexan 6-Cys gene family was originally identified in the Babesia-related Plasmodium parasites [3-5]. Members of the 6-Cys family are defined by a unique arrangement of 6-Cysteine residues, although other alternative arrangements involving 4, 5 and seven cysteine residues are also possible [2]. The members of the 6-Cys family contain signal peptides and are thus likely expressed on the surface of the parasites [2]. Interestingly, Plasmodium 6-Cys proteins such as pfs 230 and s48/45 are expressed in sexual stages of the parasite, occurring exclusively in the midgut of the mosquito vector, whereas other Plasmodium 6-Cys proteins are differentially expressed in the sporozoite and or merozoite stages [6]. Plasmodium 6-Cys proteins are known to play important roles in the sexual stage forms and are required in oocyte formation, and are strong candidates for the development of transmission-blocking vaccines [6, 7]. In addition, some Plasmodium 6-Cys proteins such as 6-Cys protein P12 and P41 are also known to be expressed in erythrocyte stages of the parasite [8]. Similar to Plasmodium parasites, B. bovis encodes for 6-Cys proteins [9]. The B. bovis 6-Cys gene family was originally described as containing six genes termed 6-Cys A-F, but, further searches of the genome revealed the presence of four additional genes (6-Cys G-J), in this family [2]. Currently, the roles of the 6-Cys gene family members in survival during the tick and mammalian stage of the life-cycle are unknown. The gene 6-Cys $\mathrm{E}$ was found transcribed only in tick stages, but not in the blood stages of parasites of the T3Bo strain [2]. In addition, previous studies performed on the biologically cloned B. bovis strain Mo7 suggested that the 6-Cys E protein might be a suitable candidate for a subunit vaccine because it was found to be expressed on the surface of the cloned B. bovis Mo7 strain, while developing in the blood stage, [9] and contained neutralization sensitive epitopes. We were interested in further analysing the biological significance of this previous finding and into determining whether expression of 6-Cys $E$ is essential for the survival of the blood stage of the life-cycle of B. bovis. Importantly, if 6-Cys $E$ mutants are viable and able to develop in in vitro cultures, it would provide an essential tool for further testing of the possible functional role of the 6-Cys $E$ gene in erythrocyte and tick stages of the life cycle of $B$. bovis.

The focus of the present study was to determine whether expression of the 6-Cys gene $E$ is needed for the replication of the blood stage of the parasite in vitro. Here, we describe the production of a B. bovis 6-Cys $E$ knockout (KO) clonal line derived from the T3Bo strain of $B$. bovis using transfection methods [9-13] that can be applied for future gene functional analysis. The B. bovis
T3Bo line was selected for this study because recent unpublished evidence suggests that the phenotypically distinct Mo7 strain, which was used in previous studies, is not likely transmissible by ticks, and therefore not suited to perform further tick transmission studies required to prove the role of 6-Cys proteins in transmission.

We also compared the ability of anti-6-Cys E antibodies to inhibit the in vitro growth of $\mathrm{KO}$ and wild-type B. bovis parasites. Collectively, the results indicated that the gene 6Cys $E$ is not essential for the development of erythrocyte stages of B. bovis. Although the results are not supportive of using the 6-Cys $\mathrm{E}$ as a blood stage vaccine component, they suggest that this protein could still be a candidate for developing transmission blocking vaccines against $B$. bovis.

\section{Methods}

\section{Parasites}

The Texas (Tx) T3Bo [14] and Mo7 strains of B. bovis were grown in long-term microaerophilic stationary phase culture as previously described [10, 11]. Both strains, Mo7 and T3Bo, of B. bovis $[10,12]$ are maintained as a cryopreserved stabilate in liquid nitrogen when not in use [13].

\section{Construction of the transfection plasmid p6-Cys-EKO}

The B. bovis E 6-Cys KO plasmid (p6-Cys-EKO, Fig. 1) was designed to express the egfp-bsd fusion protein under the control of the $e f-1 \alpha$ promoter. The egfp-bsd gene was amplified by PCR from plasmid egfp-bsd-pUC 57 plasmid containing a synthetic egfp-bsd fused gene (GenScript, Nanjing, China), using primers EGFP-FEcoRI (5'-GCT ACT GAA TTC ATG GTG AGC AAG GGC GAG-3') and Tracer-Bsd-EcoRI (5'-TAA TGT GAA TTC GCC CTC CCA CAC ATA ACC AGA G$\left.3^{\prime}\right)$. Both primers contain EcoRI restriction sites (bold fonts) to facilitate cloning into plasmid p40-15-luc [15]. Both, plasmid p40-15-luc and the PCR amplified fragment were digested with EcoRI. Restriction digestion of p40-15-luc with EcoRI results in the removal of the luciferase gene. The EcoRI-digested PCR fragment was then cloned into the EcoRI-treated plasmid p40-15-luc. The resulting plasmid, containing the ef- $1 \alpha$ promoter (Hindi III cloning site) now controlling the expression of the egfp-bsd gene (EcoRI cloning site), and the 3 ' - region of the rap-1 gene (3'-rap-1) (PstI cloning site) [16, 17], was designated p40-15-egfpbsd. To construct the p6-Cys$E K O$ plasmid, we first cloned the $5^{\prime}$ flanking region of the $E$ 6-Cys gene (fragment 5 ' flanking, Fig. 1a, b) containing $\sim 200 \mathrm{bp}$ upstream to the gene 6-Cys $E$ locus untranslated region (UTR) plus $\sim 900 \mathrm{bp}$ from the full open reading frame (orf) of the full 6-Cys $E$ gene into the XhoI site of plasmid p40-15-egfpbsd. The 5' flanking end was amplified using the forward primer (5'-GCG TGC CTC GAG GTA TTT AAC ATT ACA AAC TCC- 


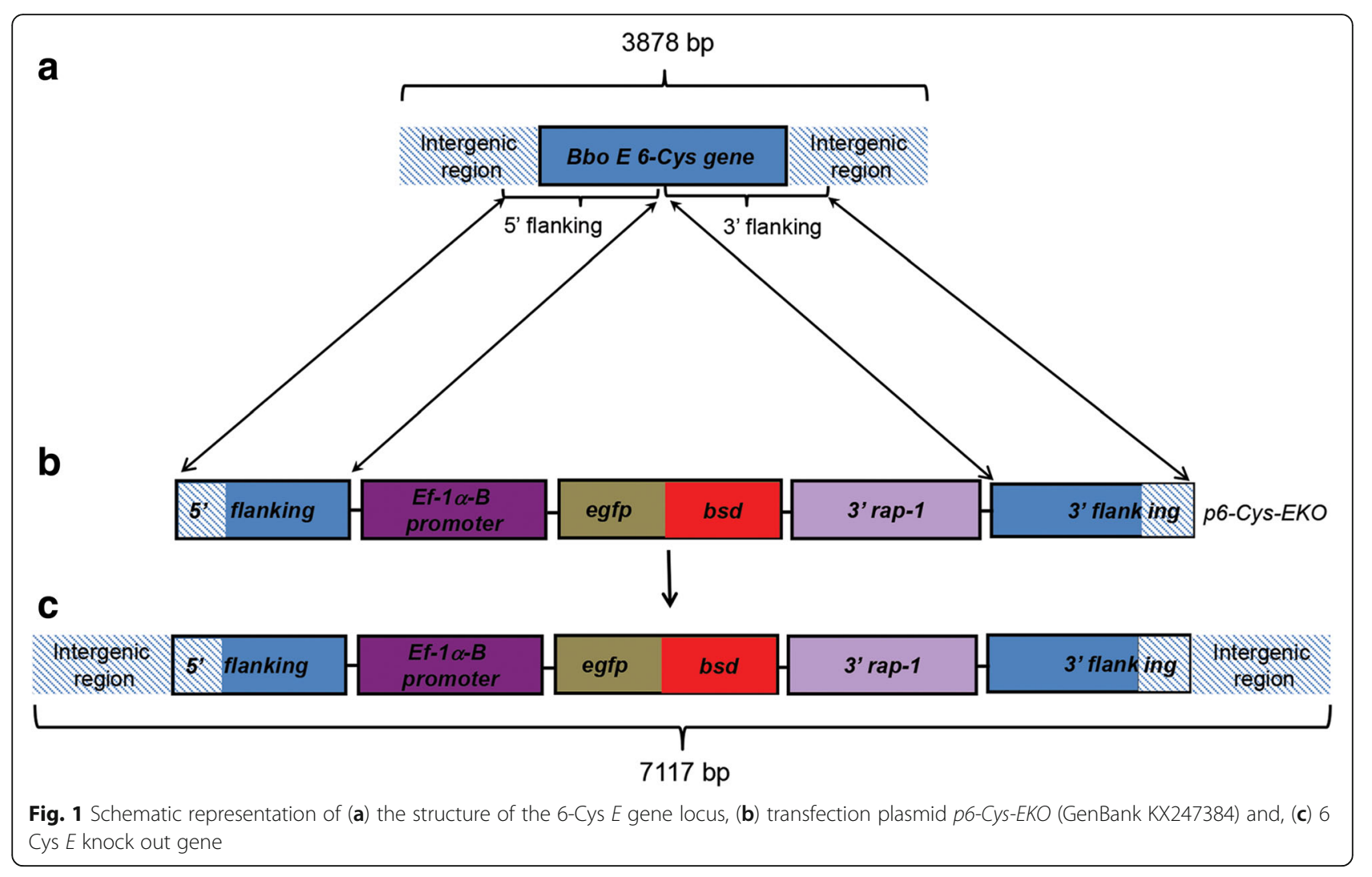

$3^{\prime}$ ) and the reverse primers (5' - GCG TGC CTC GAG CAT TGG CAT GAA AAG G-3') containing Xhol ends (bold font) for cloning into the p40-15-egfpbsd plasmid. The resulting plasmid was designated $p 40-15$-egfpbsd-5' 6-cys. The 3' 6-Cys $E$ insertion region included $\sim 870$ bp from the 3' end of the orf of $E$ 6-Cys gene with $\sim 200 \mathrm{bp}$ downstream of UTR region of E 6-Cys gene. This 3' flanking region was amplified using the forward primer is (5' -CGC TAT GGA TCC TTG ATG GTA ATA TGA GGC-3') and reverse primer (5' - CGC TAT GGA TCC CAG GGA TAG ATA ACC GG-3') containing BamHI restriction sites (bold font), and cloned into the $\mathrm{BamHI}$ site of plasmid p40-15-egfpbsd-5'6-Cys. All primers used for amplification of the 6-Cys $E$ flanking regions were designed based on genome sequence information available at http://vmp.vetmed.wsu.edu/research/apicomplexangenomics [18]. The resulting final transfection plasmid was designated $p 6-C y s-E K O$ plasmid. The p6-Cys-EKO plasmid was fully sequenced, and the assembled final sequence was deposited in GenBank (Accession number KX247384). The p6-Cys-EKO plasmid was used to transform Top10 E. coli competent cells (Invitrogen, Carlsbad, USA), and purified using Qiagen Plasmid Midi Kit (Qiagen, MD, USA) following the manufacturer's instructions prior to transfection. Plasmid control pBluescript $(p B S)$ was purified identically and used as a control in the transfection experiments.

\section{Transfection of $B$. bovis}

The plasmid $p 6-C y s-E K O$ was electroporated into B. bovis Tx T3Bo strain infected erythrocytes as previously described $[15,16]$. Plasmid pBluescript was used in identical transfections as a negative control. The transfected parasites were transferred to an incubator at $37^{\circ} \mathrm{C}$, selected with blasticidin as described previously [16], and monitored on a regular basis for the presence of transfected parasites with a fluorescence microscope at $60 \times$ amplification.

\section{Cloning transfected parasites}

Cloning was performed using a fluorescence activated cell sorter with a Clonecyte attachment (FACS Vantage, Becton Dickenson, Immunocytometry Systems, San Jose, CA) as previously described [19]. The cells were sorted depending on the fluorescence expressed from transfected parasites with the egfp gene. Single transfected parasites were sorted into three 96 well plates. The cultures were maintained for 3 weeks as described [20] with a daily change of medium. PCR analysis was performed on $1 \mu \mathrm{l}$ samples obtained from each well to identify parasites using the rap-1 primer BoFN primer ( $5^{\prime}$-TCA ACA AGG TAC TCT ATA TGG CTA CC-3') and BoRN primer (5'-CTA CCG AGC AGA ACC TTC TTC ACC AT-3') [20]. PCR positive wells were further analysed by PCR to detect a disrupted 6-Cys gene $E$ using a forward primer that anneals in the intergenic region 
upstream of 6-Cys gene $E$ IGE for (5'-GCT AAG CAC CTA TTT AGC GTA AC-3') and a reverse primer EFPr-R-6-HIII [5'-GAC CAT AAG CTT AGT AAA CGA TAG AAC AGA CTA AG-3'] [15].

\section{Phenotypic and genotypic analysis of transfected clones} Multiple approaches were used to define the phenotypic and genotypic characteristics and to confirm the integrity and stability of the transfected clones, as described below.

\section{Fluorescence microscopy}

Emission of fluorescence by transfected parasites was verified using a Zeiss Fluorescence microscope. Wells containing clones of parasites with uniform bright fluorescence ( $N=62$ clones) were selected for further analysis.

\section{$P C R$ and DNA sequencing}

PCR assays and sequencing were performed on genomic DNA (gDNA) extracted from the: i] Parental non-transfected strain, ii] B. bovis 6-Cys EKO (mixed transfected strain), and iii] B. bovis 6-Cys EKO-cln (transfected clones) to verify the integration and disruption of the 6-Cys $E$ gene by the $p 6-C y s E K O$ construct. A PCR amplifications aimed at demonstrating integration and disruption of the 6-Cys $E$ locus (Fig. 3a, b) were performed using the primers shown in Table 1. Other comparative PCR analysis was designed to detect the disruption of gene 6-Cys $E$ using a forward primer representing the genomic intergenic region upstream of gene 6-Cys $E$ named IGE for (5'-GCT AAG CAC CTA TTT AGC GTA AC-3') and the reverse primer EF-Pr-R-6-HIII (5'-GAC CAT AAG CTT AGT AAA CGA TAG AAC AGA CTA AG-3') (Fig. 3a) [15].

\section{Southern blot analysis}

The patterns of insertion of the EKO plasmid into the $B$. bovis T3Bo gDNA were analysed using hybridization of

Table 1 Primers sequence used in PCR and sequencing

\begin{tabular}{ll}
\hline Amplicon \# & Primer pair \\
\hline 1 & $\begin{array}{l}\text { For: 5'-gctaagcacctatttagcgtaac-3' } \\
\text { Rev: 5'-gaccataagcttagtaaacgatagaacagactaag-3' }\end{array}$ \\
& For: 5'-atgaagcgaaatatcgtacacaatacc-3' \\
& Rev:5'-atcgcaaagcttttcgtaaagttgcaataaattatc-3' \\
& For: 5'-ctgatcaagctttatatctgagacaacattagtatcg-3' \\
& Rev: 5'-ctacgaggatcctccttt gtgaggttcacg-3' \\
& For: 5'-gctactgaattcatggtgagcaagggcgag-3' \\
4 & Rev: 5'-ctacgaggatcctccttt gtgaggttcacg-3' \\
& For: 5'-gctactgaattcatggtgagcaagggcgag-3' \\
5 & Rev: 5'-atgtcaatagtgatcatcg-3' \\
6 & For: 5-gctactctgcaggatgagatgcgtttataatg-3' \\
& Rev: 5'-gttgcaaaaacatgttatgaag-3' \\
7 & For: 5'-ttgatggtaatatgaggc-3' \\
& Rev: 5'-gttgcaaaaacatgttatgaag-3'
\end{tabular}

undigested and BglII digested gDNA, using DIG-labeled 6-Cys E 3' end, Egfp-bsd, Ef-1 $\alpha$ and ampicillin probes in Southern blots. The probes were prepared as previously described [16]. The gDNA used in this analysis included. DNA extracted from the non-transfected parental T3Bo strain, 6-Cys EKO, and 6-Cys EKO-cln.

\section{Clonal line genome sequencing}

Approximately $7 \mu \mathrm{g}$ of gDNA were extracted from in vitro cultured B. bovis using the DNEasy kit (Qiagen, Hilden, Germany) with special care being taken to avoid shearing of large DNA fragments. The SMRT Bell Template Prep Kit V1.0 was used for library preparation, and DNA was size-selected at $>15 \mathrm{~kb}$ using the Blue Pippon (Sage Biosciences, Edmonton, Canada), the resulting library had an average fragment size of $\sim 21 \mathrm{~kb}$. Sequencing was performed on the Pacific Biosciences RSII sequencer using MagBead Loading and P6/C chemistry. Sequences were assembled using the Hierarchal Genome Assembly Process 2 (HGAP2), and the resulting assembly was further analysed using CLC Genomics Workbench. Comparison of the re-sequencing data from the transfected clone to that of the T2Bo reference genome was performed using BLAT 2.0 using default settings [21].

\section{Phenotypic analysis of mutant strain and clone}

To identify the possible impact of the gene 6-Cys $E$ mutation on the growth of the B. bovis parasite, we compared the ability of the 6-Cys EKO strain, 6-Cys EKO-cln and wild-type T3Bo strains to grow in in vitro culture. Cultures of each strain were initiated at $0.5 \%$ parasitemia in triplicate wells in the presence and absence of blasticidin [16]. Culture medium was replaced and the parasitemia calculated daily for 8 days. Statistical analysis was performed with the Student $t$-test, and the probability value of less than $5 \%(P<0.05)$ was considered significant.

\section{In vitro neutralisation assay}

Inhibition of $B$. bovis merozoite invasion of erythrocytes was performed on the Mo7, T3Bo, EKO, and EKO cln, strains as previously described by Hines et al. [22]. Briefly, B. bovis merozoites were separated from erythrocytes by centrifugation, and approximately $5 \times 10^{5}$ viable merozoites, as determined using 6-carboxyl fluorescein diacetate [23], were used in each antibody neutralisation reaction: rabbit antisera specific for B. bovis 6-Cys E-peptides 1 and 2 [22], or pre-immune rabbit sera. Mouse polyclonal sera against a non-Babesia sp. protein (sera specific for Operon-associated protein (OpAG) 2 encoded protein of A. marginal) [9] was used as negative control, and a previously described $B$. bovis neutralising monoclonal mouse anti-MSA-1 antibody (BABB35A4) was used as positive control [22, 24]. All sera were heat-inactivated for $30 \mathrm{~min}$ at $56{ }^{\circ} \mathrm{C}$, diluted $1: 1$ in culture medium and incubated 
with the merozoites for $30 \mathrm{~min}$ at $4{ }^{\circ} \mathrm{C}$. Mouse antibodies were used at a concentration of $1.5 \mu \mathrm{g} / \mathrm{ml}$. After the incubation period, an equal volume of $5 \%(\mathrm{v} / \mathrm{v})$ bovine erythrocytes in culture medium was added to each well. The plates were incubated at $37{ }^{\circ} \mathrm{C}$ in a $5 \% \mathrm{CO}_{2}$ atmosphere. Parasites were grown in micro-aerophilous stationary phase culture as previously described [11]. Percentages of parasitized erythrocytes (PPE) were determined every $24 \mathrm{~h}$ up to $72 \mathrm{~h}$ by counting parasites in smears stained with Diff-Quik on an optical microscopic. The assay was performed in triplicate in 96-well plates. The results were analyzed by the one-way ANOVA with 95\% confidence level.

\section{Results and discussion}

Transfection and biological cloning of transfected B. bovis parasites

Parasites of the B. bovis T3Bo strain were electroporated with plasmid p6-Cys-EKO (GenBank KX247384). Plasmid $p 6-C y s-E K O$ contains $5^{\prime}$ and $3^{\prime}$ flanking regions designed for disrupting the structure of the 6-Cys gene $E$ upon insertion of the egfp-bsd selectable marker in the $B$. bovis genome by homologous recombination. Schematic representations of plasmid $p 6-C y s-E K O$, the target locus, and the expected structure of the 6-Cys gene $E$ in $\mathrm{KO}$ parasites, are shown in Fig. 1. Electroporated parasites were selected in in vitro cultures with inhibitory doses of blasticidin [16] starting $4 \mathrm{~h}$ after electroporation, and a blasticidin-resistant and egfp fluorescent $B$. bovis line termed $B$. bovis 6-Cys EKO emerged 7 days upon the start of blasticidin selection. In contrast, no parasites transfected with the pBluescript plasmid were detected in identically blasticidin-treated cultures at day seven upon the start of selection (Fig. 2). The transfected fluorescent parasite line was grown in in vitro cultures for additional 30 days in the presence of inhibitory concentrations of blasticidin before it was expanded for genotypic analysis and biological cloning. The genotypic analysis of the 6-Cys EKO parasite lines is described below. A clonal transfected $B$. bovis line containing a disrupted 6-Cys gene $E$ was then derived from the parasite line B. bovis 6-Cys EKO using FACS sorting. FACS sorted single-parasite cells were grown in in vitro cultures developed in a low $\mathrm{O}_{2}$ incubator set at $37{ }^{\circ} \mathrm{C}$. The cultured parasites were then screened for the presence of a disrupted 6-Cys gene $E$ by PCR. A fluorescent clonal parasite line containing the egfp-bsd gene termed $B$. bovis 6-Cys EKO-cln emerged in the in vitro cultures 3 weeks after the onset of the FACS cloning. The experiments described below demonstrate the transfected genes effectively disrupt the integrity of the 6-Cys gene $E$ in line 6-Cys EKO-cln.

\section{Genotypic analysis of transfected $B$. bovis parasites}

We first performed PCR to characterise the disrupted 6Cys gene $E$ locus in parasites of the line 6-Cys EKO-cln. Seven sets of PCR primers were designed to demonstrate disruption of the 6-Cys gene $E$ in transfected parasites (Table 1 and Fig. 3a). The results of the seven distinct PCR reactions performed on gDNA extracted from the 6-Cys $\mathrm{KO}-\mathrm{cln}$ are shown in Fig. 3a. Size and sequence comparison of the PCR products are consistent with the disruption of the targeted 6-Cys gene $E$ and "in target" insertion of the transfected genes in the 6-Cys EKO-cln parasites.

To further confirm specific disruption of the targeted 6-Cys $E$ locus, we also performed comparative PCR among the T3Bo, 6-Cys EKO, and 6-Cys EKO-cln using primers designed for the amplification of rap-1 (control non-targeted gene), full-size 6-Cys gene $E$, and egfp-bsd.

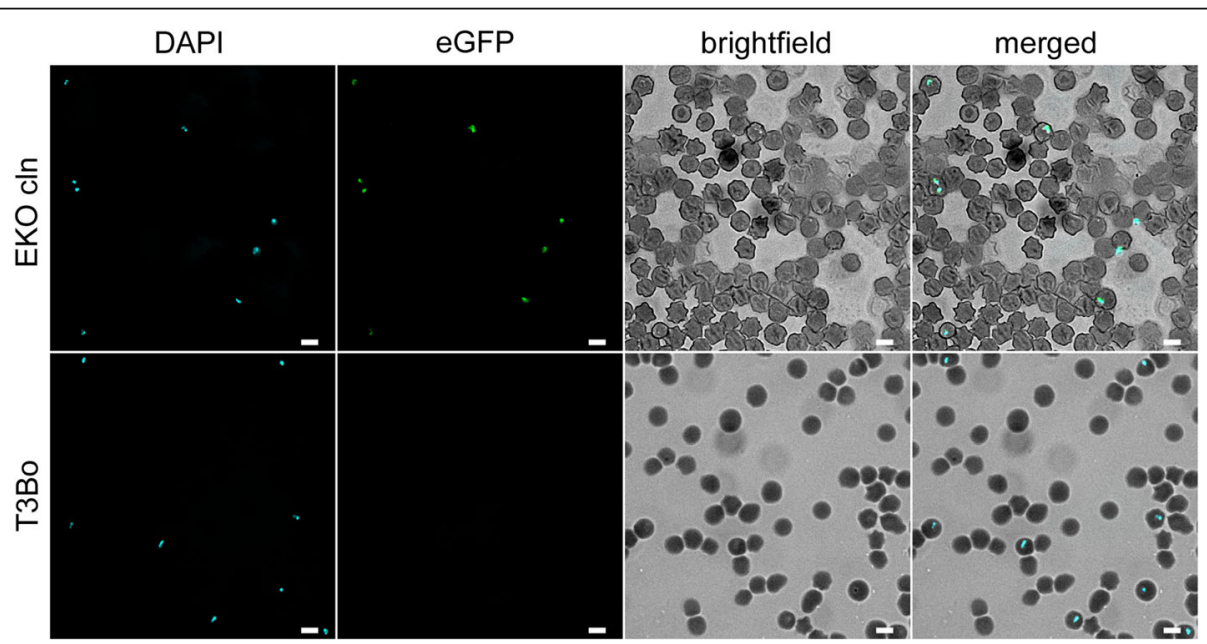

Fig. 2 Detection of the expression of egfp in B. bovis 6-Cys EKO mutant line by fluorescence microscopy. Upper panels: (left to right) represents transfected parasites of the EKO CIn line stained with DAPI, fluorescent light, brightfield and a merged image respectively. Lower panel identical images obtained using the control non-transfected parental parasite line T3Bo. Scale-bars: $5 \mu \mathrm{m}$ 


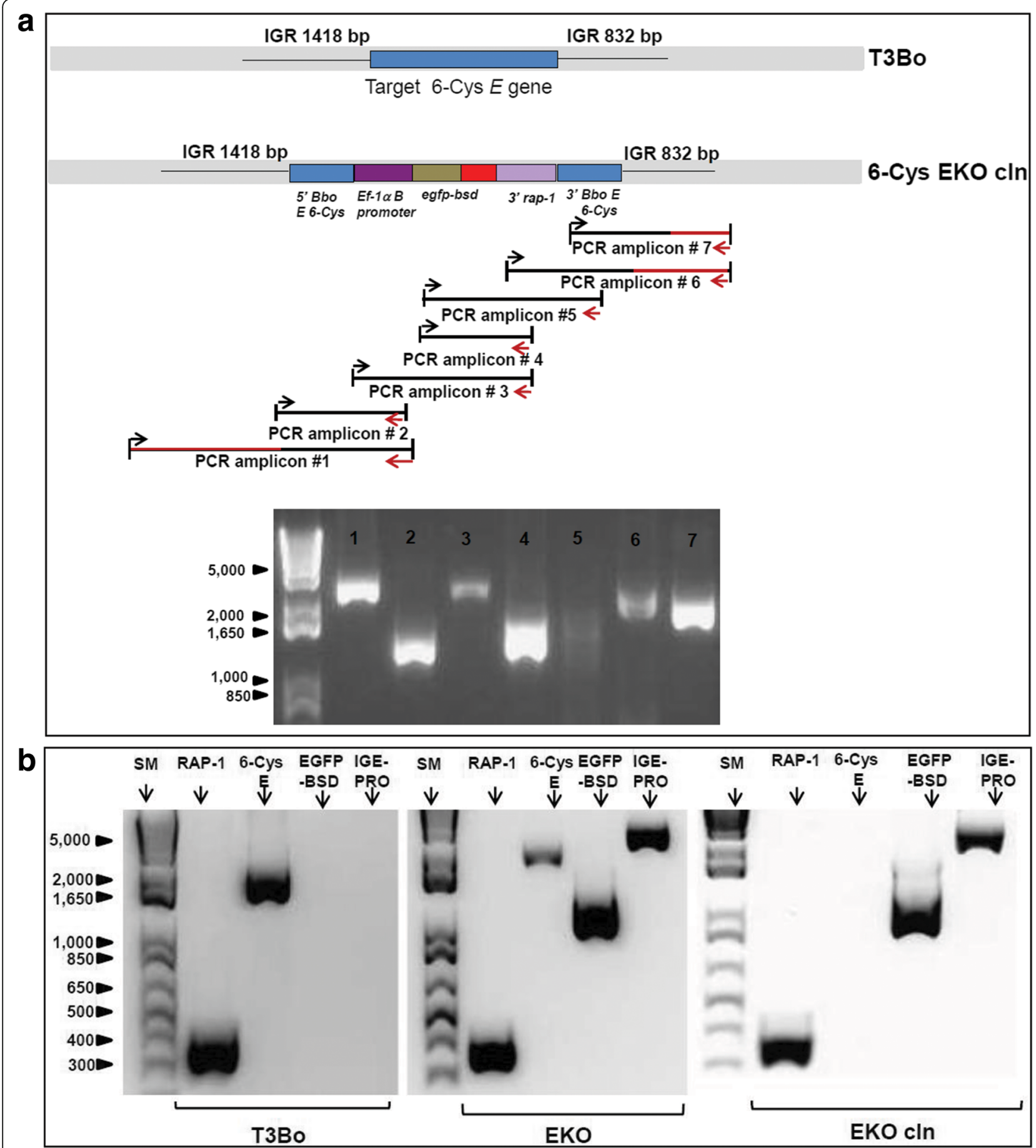

Fig. 3 PCR analysis for integration of egfp-bsd in transfected B. bovis. a Top panel: schematic representation of the intact 6-Cys $E$ locus in the T3Bo strain and the 6-Cys EKO-CIn parasites as deduced from full genome sequence of 6-Cys EKO-Cln parasites. The red coloured lines represent gDNA sequences that are not a part of the targeted region included in the transfection plasmid p6-Cys-EKO. Lower panel: agarose electrophoresis analysis performed on the PCR products corresponding to each of the fragments numbers 1-7 in the upper panel. $\mathbf{b}$ Comparative PCR analysis performed on the line EKO-CIn, T3Bo, and EKO, using RAP-1, 6-Cys E, egfpbsd, and IGE-Pro primer sets. SM represents standard molecular weight 10 Kbp

Additionally, to demonstrate specific integration at the attempted 6-Cys $E$ locus, we also performed a PCR reaction using forward primers IGE (representing sequences in the intergenic region located $5^{\prime}$ of the $E$ gene, and not represented in the transfection plasmid $p 6-C y s-E K O)$ and $\mathrm{PRO}$ (representing sequences in the ef-1B promoter) 
as the reverse primer (Table 1 and Fig. $3 \mathrm{~b}$ ). All products derived from these PCR reactions, shown in Fig. 3b were fully sequenced. The control rap-1 gene was identically amplified from gDNA extracted from all three strains. However, gDNA from strains 6-Cys EKO and 6-Cys EKO-cln, but not from the T3Bo strain, generated products when the egfp-bsd and IGE-PRO primers were employed, confirming the presence of the egfp-bsd gene and integration of transfected genes into the 6-Cys gene $E$ locus exclusively in the transfected parasites. Furthermore, sequence analysis of PCR amplicons indicates the integration of the structure of the locus by sequences present in the transfected plasmid, which successfully integrated into the genome by homologous recombination. Interestingly, primers designed for the amplification of the full size 6-Cys $E$ orf only generated PCR products of identical size and composition in the T3Bo and 6-Cys EKO lines, and no products were visible upon amplification of the 6-Cys EKO-cln line, consistent with disruption of the 6-Cys gene $E$ in these parasites (Fig. 3b). Taken together, these data also suggest that the transfected line 6-Cys EKO contains a mixed population of parasites, either with or without disrupted 6-Cys gene $E$, and that, in contrast, the 6-Cys EKO-cln line contains a single insertion of the egfp-bsd gene disrupting the integrity of the 6-Cys gene $E$ of B. bovis.

Total gDNA extracted from cultured wild-type T3Bo, 6-Cys EKO and 6-Cys EKO-cln parasites was then analysed by Southern blot analysis (Fig. 4). The gDNA was digested with BglII which does not cut inside the $p 6$ -
Cys-EKO plasmid and the 6-Cys $E$ locus, separated by agarose gel electrophoresis, and transferred to blotting nylon membranes. The blots were hybridised with diglabeled specific egfp-bsd, ef- $1 \alpha$, and ampicillin (plasmid $p B S$ marker) probes (Fig. 4a-c, respectively). Taken together, the Southern blot analysis indicated that: (i) both the mixed transfected and clonal strain, but not the wild-type non-transfected, parasites contain a single copy of the egfp-bsd gene; (ii) the clonal 6-Cys KO-cln hybridize with 6-Cys $3^{\prime}$ probe in a different pattern compared to the wild type and mixed transfected line, suggesting that these parasites contain a disrupted 6-Cys gene $E$; (iii) the ampicillin probe hybridizes with transfection plasmid likely present in the cells as episomal DNA, present only in the mixed line. Plasmid DNA was not detected either in the wild type or the 6-Cys EKO$c l n$; (iv) the patterns of hybridization of the egfp-bsd probe suggests that the mixed transfected line 6-Cys $E K O$ contains either multiple egfp-bsd copies inserted in the genome, or, distinct subpopulations of transfected parasites including non-specific insertions; Yet, the results may also be due to the lasting presence of single or concatenated episomal transfection plasmid (as shown in Fig. 4c) in the transfected parasites of this line; (v) the ef- $1 \alpha$ probe hybridized with two bands in the EKO-cln line, a $\sim 12 \mathrm{~kb}$ band corresponding to the intact ef-1 $\alpha$ locus, and an upper $(\sim 23 \mathrm{~kb})$ band which is identical is size with the fragment hybridizing with the egfp probe (Fig. 4a). These patterns of hybridization were expected and are likely due to the presence of the sequence in the
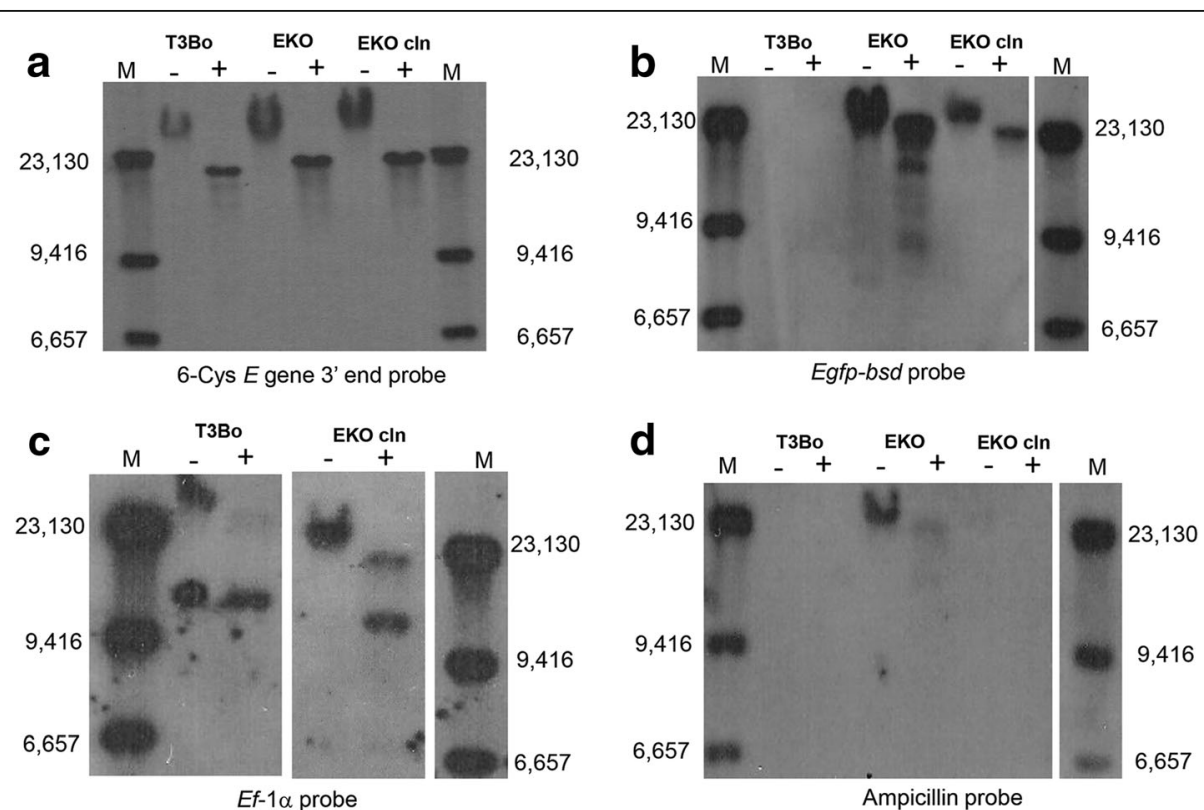

Fig. 4 Southern blot analysis of gDNA extracted from non-transfected T3Bo,6-Cys EKO, and 6-CysEKO-cln, using Dig-labeled probes against 6-Cys E gene $3^{\prime}$ end probe (a), egfp-bsd probe (b), Ef-a probe (c) and ampicillin probe (d). Each sample was analysed using undigested (-), or Bglll digested (+) gDNA. M: molecular marker 
ef- $1 \alpha$ probe into the transfection construct that integrated into the 6 Cys locus.

Collectively, this Southern blot and PCR data support stable insertion of the intended egfp-bsd gene disrupting the integrity of the 6-Cys gene $E$ orf in in the parasite line 6-Cys EKO-cln, as operated by homologous recombination. Full genome sequencing of the 6-Cys EKO-cln line again confirmed the gene 6-Cys $E$ interruption and is consistent with the PCR and Southern blot data. Sequence analysis of chromosome number 2, where the 6Cys gene $E$ locus is located indicates that the originally 3878 bp targeted area became enlarged to $7117 \mathrm{bp}$ as a result of the integration of the egfp-bsd gene, its 3 ' rap and the $e f-1 \alpha$ B promoter regions included in the transfection, interrupting the 6-Cys gene $E$, exactly as described in Figs. 1 and 3a. The pattern of insertion and the conservation of restriction sites derived from the plasmid vector are shown in Additional file 1: Figure S1. The sequence of the interrupted 6-Cys gene $E$ region in the 6-Cys E-KO-cln parasites was deposited in GenBank (Accession no. KX247383). Analysis of the full genome of the 6-Cys EKO-cln gDNA indicated that no other insertions derived from the transfection changes occurred in the genome of parasites of the 6-Cys EKO-cln line. Comparison of the original reference $B$. bovis T2Bo genome sequence [18] to the re-sequenced 6-Cys EKO-cln revealed random SNP and short insertion/deletion mutations but did not result in gross chromosomal re-arrangements. However, such minor differences among these genomes are expected as they result from comparing sequences derived from a clonal parasite line with a parasite line composed of multiple parasite subpopulations.

\section{Phenotypic characterization of $B$. bovis transfected parasites}

The patterns and rates of in vitro growth among the wild type T3Bo, 6-Cys EKO, and 6-Cys EKO-cln strains were compared. No significant differences $(P<0.05)$ were found in the rate of the in vitro growth among these three parasite lines, regardless of the presence or absence of the selective marker blasticidin in the culture medium (Fig. 5). We concluded from these experiments
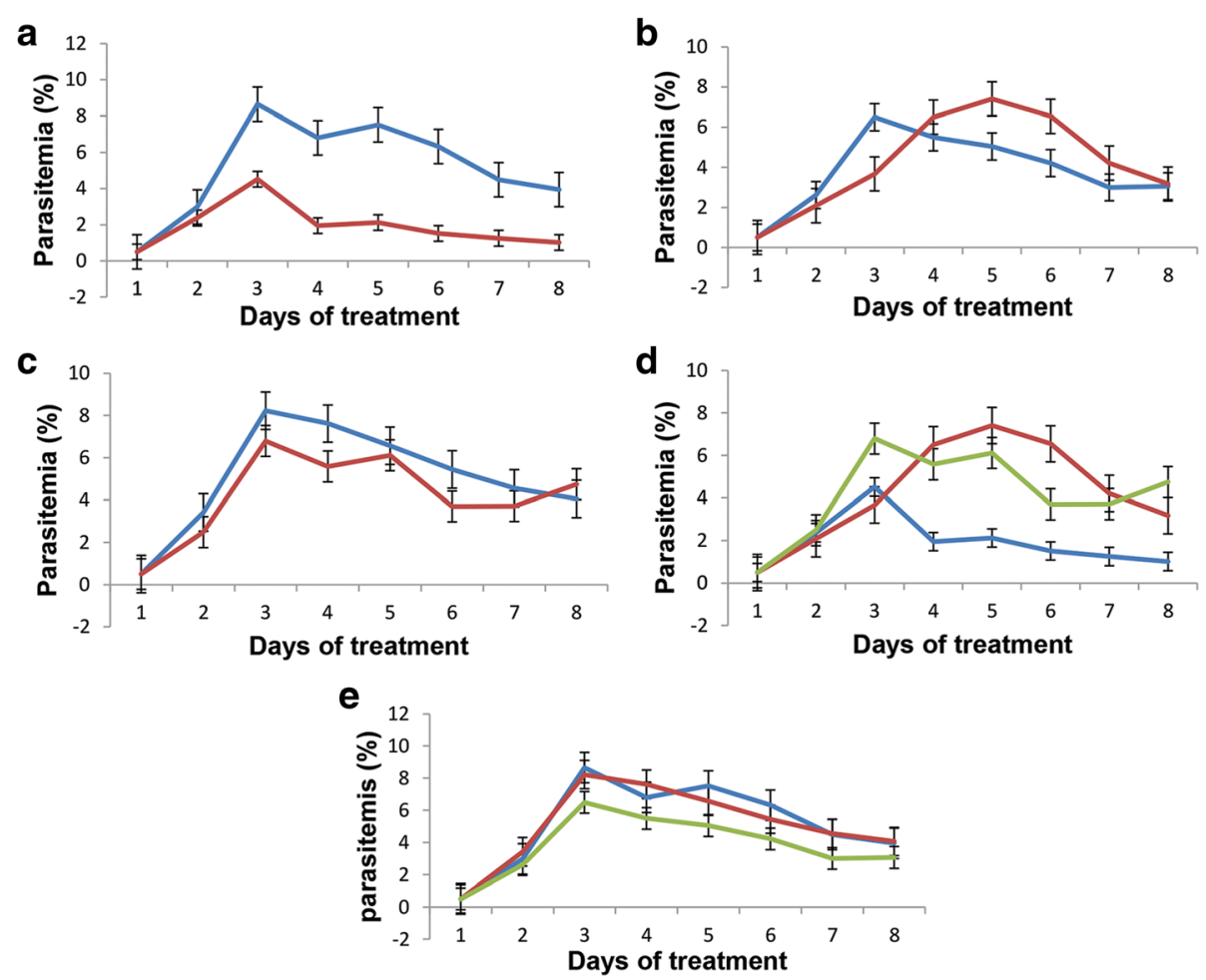

Fig. 5 Comparative in vitro culture growth curves of B. bovis T3Bo, transfected 6-Cys EKO, and 6-Cys EKO-Cln parasite lines with and without addition of inhibitory doses of blasticidin. The data are expressed as arithmetic means \pm standard deviation (SD). $P<0.05$ indicates a statistically significant difference and $P>0.05$ indicating a statistically no significant difference. a In vitro culture growth curves of T3Bo strain without (blue line) and with the addition of blasticidin (red line). ( $\left.t_{(12)}=4.1, P=0.0013\right)$. $\mathbf{b}$ In vitro culture growth curves of the 6-Cys EKO without (blue line), and with blasticidin addition (red line) $\left(t_{(12)}=-0.55, P=0.58\right)$. $\mathbf{c}$ In vitro culture growth curves of 6-Cys EKO-cIn line without (blue line) or with blasticidin addition (red line) ( $t_{(12)}=0.87$, $P=0.399$ ). $\mathbf{d}$ In vitro culture growth curves of T3Bo (blue line), 6-Cys EKO strain (red line), and 6-Cys EKO-cln line (green line) with blasticidin addition. Statistical comparisons: T3Bo vs 6-Cys EKO lines ( $\left.t_{(12)}=3.1, P=0.009\right)$; T3Bo vs 6 -Cys EKO- $\ln$ line $\left(t_{(12)}=3.6, P=0.003\right)$. e In vitro culture growth curves of T3Bo strain (blue line), 6-Cys EKO (red line) and 6-Cys EKO-cln lines (green line) without blasticidin addition. Statistical comparisons: T3Bo vs 6-Cys EKO lines $\left(t_{(12)}=-1.6, P=0.13\right) ;$ T3Bo vs 6-Cys EKO-CIn line $\left(t_{(12)}=-0.25, P=0.80\right)$ 
that a mutation affecting the expression of the 6-Cys $E$ gene in the line 6-Cys EKO-cln does not affect the fitness of the erythrocyte stages of B. bovis parasites while developing in in vitro cultures. In addition, the data demonstrate that the 6-Cys EKO-cln parasites can develop similarly in in vitro cultures regardless of the presence or absence of the selective pressure provided by blasticidin. These results suggest that expression of the 6-Cys gene $E$ in erythrocyte stages is not essential for the development of B. bovis merozoites in in vitro cultures, and suggest that this protein is no longer a viable target for blood-stage vaccine development. However, because it is expressed in the tick stages of the tick, the 6-Cys E protein remains as a potential candidate for the development of transmission-blocking vaccines.

Previous studies demonstrated surface expression of the 6-Cys gene $E$ in the clonal line Mo7. However, identical Western blot analysis performed on T3Bo and 6-Cys EKO parasites using rabbit antibodies against synthetic peptides representing B-cell epitopes in 6-Cys protein E failed in reacting with any parasite product in the T3Bo and 6-Cys EKO parasites (data not shown). In addition, we previously demonstrated that rabbit antibodies directed against synthetic peptides derived from 6-Cys gene $E$ [9] have an inhibitory effect on the growth of B. bovis Mo7 parasites, suggesting the functional relevance of this protein in erythrocyte stages. An identical in vitro blocking assay [9] was performed in this study on parasites of the Mo7, T3Bo, 6-Cys EKO and 6-Cys EKO-cln lines using rabbit pre-immune serum, rabbit anti 6-Cys $E$, monoclonal anti-B. bovis MSA-1, and non-related B. bovis monoclonal antibodies (A. marginale OpAG2). The results of the experiment are shown in Fig. 6, and ANOVA statistical analysis in Additional file 2: Table S1. As expected, the control anti-MSA-1 monoclonal antibodies almost completely abrogate the growth of the parasites at $72 \mathrm{~h}$ in all $B$. bovis strains tested. Also, and fully consistent with previously reported data, the rabbit antibodies against the 6-Cys $E$ protein [9] are able to inhibit the growth of Mo7 and T3Bo parasites when compared to the pre-immune rabbit sera $\left(F_{(2,6)}=20.55, P=0.002 ; F_{(2,6)}=6.83, P=0.028\right.$, for Mo7 and T3Bo, respectively) (Additional file 2: Table S1) $72 \mathrm{~h}$ after the addition of the antibodies, although the levels of inhibition are much lower compared to anti-
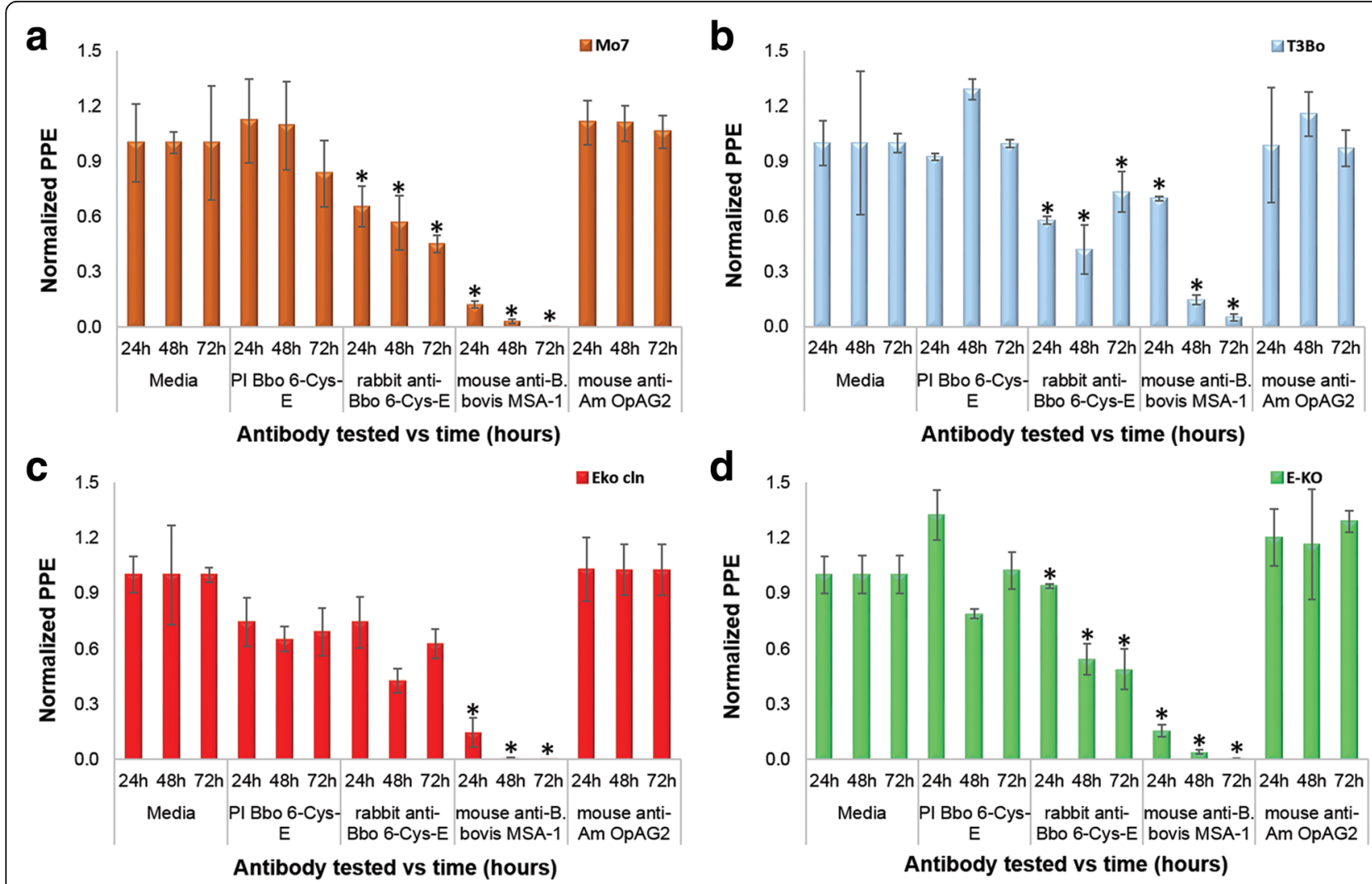

Fig. 6 In vitro neutralisation assay performed on Mo7 strain (a), T3Bo strain (b), EKO- cln (c), and EKO (d). Normalised PPE values (Y axis) obtained from B. bovis culture at $72 \mathrm{~h}$ in the presence or absence of different sera as indicated on the X-axis. Error bars indicate standard deviations for each sample tested from triplicate culture. Data from each B. bovis strain tested, and data from each treatment (pre-immune Bbo 6-Cys $E$ and rabbit anti-Bbo 6-Cys-E) were compared using ANOVA analysis. $\left(^{*}\right)$ represent $P<0.05$ indicating a statistically significant difference between groups 
MSA-1 antibody. However, and consistent with the lack of the 6-Cys $E$ gene in these parasites, these antibodies have negligible inhibitory activity on the 6-Cys EKO-cln parasites $\left(F_{(2,6)}=4.90, P=0.06\right)$ (Additional file 2: Table S1) and a significant inhibitory effect on the growth of the 6Cys EKO line $\left(F_{(2,6)}=37.02, P=0.000\right)$ (Fig. 6 and Additional file 2: Table S1). Marginal inhibition of the 6-Cys EKO line might be due to the presence of B. bovis transfected subpopulations in this parasite line that are resistant to blasticidin, but lack a mutation in the 6-Cys $E$ gene. Thus, overall, these observations also suggest that, at least under the culture conditions tested in this study, the level of expression of the 6-Cys gene $E$ in blood stages is likely decreased compared to the T3Bo parasite line, in contrast to the Mo7 clonal parasite line. It can be hypothesized that the low levels of wild-type B. bovis inhibition using anti 6-Cys $\mathrm{E}$ antibodies, compared to almost full inhibition caused by the MSA-1 antibody observed in Mo7, and $\mathrm{T} 3 \mathrm{~B}$ parasites might be due either to low levels or total lack of expression of the 6-Cys $E$ in a significant subpopulation of parasites growing in in vitro cultures. This interpretation is consistent with the low levels of 6-Cys $E$ transcripts detected in RNA seq experiments [25]. Moreover, the in vitro neutralisation results are consistent with the lack of expression of the 6-Cys gene $E$ in the EKO-cln, and thus this clonal line should be suitable for assessing the role of the B. bovis 6-Cys gene $E$ in the transmission of the parasite by ticks in future experiments. In summary, sequence analysis of the genome of the EKO-cln demonstrates effective disruption of the 6 Cys $E$ gene in this line. Therefore, it is doubtful that EKO-cln parasites can express a full-size version of the protein. However, the possibility remains for expression of a truncated 6-Cys $E$ protein, but this is unlikely since the necessary 3 ' canonical signals required for RNA processing (such as the poly-A tail) and translation are no longer present in the truncated gene. Another conclusion derived from the phenotypic characteristics of the 6-Cys EKO-cln line is that the partial growth inhibition observed in the Mo7 parasites must be likely due to steric hindrance caused by the binding of antibodies to a surface exposed antigen, rather than to a functional requirement for the 6-Cys E protein for the growth of blood stage parasites. This possibility is supported by the observation that parasites unable to express the 6-Cys $E$ gene are still able to grow at a similar rate as 6-Cys gene $E$ intact parasites in in vitro cultures. Future experiments will be aimed at testing whether 6-Cys $E$ mutated parasites can complete the parasite life cycle inside its tick vector.

\section{Conclusions}

In the current study, we were able to produce the clonal line 6-Cys EKO cln, and demonstrate that this clonal line contain a disruption of the B. bovis 6-Cys gene $E$.
The egfp-bsd fusion gene insertion in the clonal line 6Cys EKO cln seem to be limited to the intended 6-Cys gene $E$, and transfection does not appear to have altered the genome of 6-Cys EKO $c l n$ at sites other than that the targeted 6-Cys $E$ gene, nor causing any gross chromosomal re-arrangements. The 6-Cys EKO cln mutant line has neither obvious morphological nor significant phenotypical differences compared to the parental non-transfected strain T3Bo parasites, and it can grow at identical rates regardless of the presence or absence of the selection agent blasticidin. Moreover, 6-Cys EKO $c l n$ can resist the blocking inhibitory effects of antibodies against 6-Cys E. Taken together, the data indicate a negligible role of the 6-Cys E protein for the development of $B$. bovis parasite in in vitro cultures. We plan next to compare transmission fitness of the 6-Cys EKO $\mathrm{cln}$ and 6-Cys intact parasites to start assessing the possible role of the 6-Cys $E$ in the transmission of B. bovis.

\section{Additional files}

Additional file 1: Figure S1. Simplified restriction enzyme site map of transfection plasmid p6-Cys-EKO and in the disrupted 6-Cys locus of 6-Cys EKO-cln line, upon integration through homologous recombination. The map was deduced from full plasmid and genome sequencing of p6-Cys-EKO and 6-Cys EKO-cln parasites, respectively. (TIF $211 \mathrm{~kb}$ )

Additional file 2: Table 2. Statistical analysis of the in vitro neutralisation assay for the four B. bovis strains tested at $72 \mathrm{~h}$. a Represents the Mo7 strain, b the T3Bo strain, $c$ the EKO-cIn line; and, $d$ the EKO line. Sig. represents statistical significance at $* P<0.05$. (DOCX $17 \mathrm{~kb})$

\section{Acknowledgments}

We are thankful to Paul lacy, Jacob Laughery and Grace Chung for excellent technical support.

\section{Funding}

This work was supported by scholarship from Egyptian government, the Ministry of Higher Education and Scientific Research, and the United States Department of Agriculture Research Service Current Research Information System Project No. 2090-32000-039-00D.

\section{Availability of data and materials}

All data generated or analyzed in this study are included and its Additional file 1: Figure S1 and Additional file 2: Table S1. Sequences were submitted to GenBank database under accession numbers KX247384 and KX247383.

\section{Authors' contributions \\ Project designing and manuscript preparations CES and HFA; sample collection HFA; data acquisition: HFA, WCD, DAS, MGS, CES and DRH. All authors read and approved the final manuscript.}

\section{Competing interests}

The authors declare that they have no competing interests.

\section{Consent for publication}

Not applicable.

Ethics approval and consent to participate Not applicable.

\section{Publisher's Note}

Springer Nature remains neutral with regard to jurisdictional claims in published maps and institutional affiliations. 


\section{Author details}

'Department of Veterinary Microbiology and Pathology, College of Veterinary Medicine, Washington State University, Pullman, WA, USA. ${ }^{2}$ Parasitology and Animal Diseases Department, National Research Center, Dokki, Giza, Egypt.

${ }^{3}$ Animal Disease Research Unit, United States Department of Agriculture Agricultural Research Service, Pullman, WA, USA.

Received: 25 October 2016 Accepted: 19 April 2017

Published online: 02 May 2017

\section{References}

1. Florin-Christensen M, Suarez CE, Rodriguez AE, Flores DA, Schnittger $L$. Vaccines against bovine babesiosis: where we are now and possible roads ahead. Parasitology. 2014;141(12):1563-92.

2. Alzan HF, Lau AO, Knowles DP, Herndon DR, Ueti MW, Scoles GA, et al. Expression of 6-Cys Gene Superfamily Defines Babesia bovis Sexual Stage Development within Rhipicephalus microplus. PLoS One. 2016;11(9):e0163791.

3. Williamson KC, Criscio MD, Kaslow DC. Cloning and expression of the gene for Plasmodium falciparum transmission-blocking target antigen, Pfs230. Mol Biochem Parasitol. 1993;58(2):355-8.

4. Pradel G. Proteins of the malaria parasite sexual stages: expression, function and potential for transmission blocking strategies. Parasitology. 2007; 134(14):1911-29.

5. van Dijk MR, Janse CJ, Thompson J, Waters AP, Braks JA, Dodemont HJ, et al. A central role for P48/45 in malaria parasite male gamete fertility. Cell. 2001:104(1):153-64.

6. Van Dijk MR, Van Schaijk BC, Khan SM, Van Dooren MW, Ramesar J, Kaczanowski $\mathrm{S}$, et al. Three members of the 6-cys protein family of Plasmodium play a role in gamete fertility. PLoS Pathog. 2010;6(4):e1000853.

7. Tonkin ML, Arredondo SA, Loveless BC, Serpa JJ, Makepeace KA, Sundar N, et al. Structural and biochemical characterization of Plasmodium falciparum 12 (Pf12) reveals a unique interdomain organization and the potential for an antiparallel arrangement with Pf41. J Biol Chem. 2013;288(18):12805-17.

8. Taechalertpaisarn T, Crosnier C, Bartholdson SJ, Hodder AN, Thompson J, Bustamante LY, et al. Biochemical and functional analysis of two Plasmodium falciparum blood-stage 6-cys proteins: P12 and P41. PLoS One. 2012;7(7):e41937.

9. Silva MG, Ueti MW, Norimine J, Florin-Christensen M, Bastos RG, Goff WL, et al. Babesia bovis expresses Bbo-6cys-E, a member of a novel gene family that is homologous to the 6-cys family of Plasmodium. Parasitol Int. 2011;60(1):13-8.

10. Hines SA, McElwain TF, Buening GM, Palmer GH. Molecular characterization of Babesia bovis merozoite surface proteins bearing epitopes immunodominant in protected cattle. Mol Biochem Parasito. 1989;37(1):1-9.

11. Levy MG, Ristic M. Babesia bovis: continuous cultivation in a microaerophilous stationary phase culture. Science. 1980;207(4436):1218-20.

12. Rodriguez SD, Buening GM, Green TJ, Carson C. Cloning of Babesia bovis by in vitro cultivation. Infect Immun. 1983;42(1):15-8.

13. Palmer DA, Buening GM, Carson CA. Cryopreservation of Babesia bovis for in vitro cultivation. Parasitology. 1982:84(Pt 3):567-72.

14. Goff W, Johnson WC, Cluff C. Babesia bovis Immunity: In Vitro and in Vivo Evidence for IL-10 Regulation of IFN- $y$ and iNOSa. Ann NY Acad Sci. 1998; 849(1):161-80

15. Suarez CE, Norimine J, Lacy P, McElwain TF. Characterization and gene expression of Babesia bovis elongation factor-1a. Int J Parasitol. 2006;36(8):965-73.

16. Suarez CE, McElwain TF. Stable expression of a GFP-BSD fusion protein in Babesia bovis merozoites. Int J Parasitol. 2009;39(3):289-97.

17. Suarez CE, McElwain TF. Transfection systems for Babesia bovis: a review of methods for the transient and stable expression of exogenous genes. Vet Parasitol. 2010;167(2):205-15.

18. Brayton KA, Lau AO, Herndon DR, Hannick L, Kappmeyer LS, Berens SJ, et al. Genome sequence of Babesia bovis and comparative analysis of apicomplexan hemoprotozoa. PLoS Pathog. 2007;3(10):1401-13. doi:10.1371/journal.ppat.0030148.

19. Suarez CE, Johnson WC, Herndon DR, Laughery JM, Davis WC. Integration of a transfected gene into the genome of Babesia bovis occurs by legitimate homologous recombination mechanisms. Mol Biochem Parasitol. 2015; 202(2):23-8. doi:10.1016/j.molbiopara.2015.09.003.

20. Suarez CE, Laughery JM, Schneider DA, Sondgeroth KS, McElwain TF. Acute and persistent infection by a transfected $\mathrm{Mo} 7$ strain of Babesia bovis. Mol Biochem Parasitol. 2012;185(1):52-7.

21. Kent WJ. BLAT - the BLAST-like alignment tool. Genome research. 2002;12(4): 656-64. doi: 10.1101/gr.229202. Article published online before March 2002.
22. Hines SA, Palmer GH, Jasmer DP, McGuire TC, McElwain TF. Neutralizationsensitive merozoite surface antigens of Babesia bovis encoded by members of a polymorphic gene family. Mol Biochem Parasitol. 1992;55(1-2):85-94.

23. McElwain T, Perryman L, Davis W, McGuire T. Antibodies define multiple proteins with epitopes exposed on the surface of live Babesia bigemina merozoites. J Immunol. 1987;138(7):2298-304.

24. Suarez CE, Florin-Christensen M, Hines SA, Palmer GH, Brown WC, McElwain TF. Characterization of allelic variation in the Babesia bovis merozoite surface antigen 1 (MSA-1) locus and identification of a cross-reactive inhibitionsensitive MSA-1 epitope. Infect Immun. 2000;68(12):6865-70.

25. Pedroni MJ, Sondgeroth KS, Gallego-Lopez GM, Echaide I, Lau AO. Comparative transcriptome analysis of geographically distinct virulent and attenuated Babesia bovis strains reveals similar gene expression changes through attenuation. BMC Genomics. 2013;14(1):1.

\section{Submit your next manuscript to BioMed Central and we will help you at every step:}

- We accept pre-submission inquiries

- Our selector tool helps you to find the most relevant journal

- We provide round the clock customer support

- Convenient online submission

- Thorough peer review

- Inclusion in PubMed and all major indexing services

- Maximum visibility for your research

Submit your manuscript at www.biomedcentral.com/submit
) Biomed Central 